\title{
Long-term memory following serial discrimination reversal learning*
}

\author{
WILLIAM H. CALHOUN \\ and \\ GEORGE W. HANDLEY \\ University of Tennessee, Knoxville, Tenn. 37916
}

Twelve rats were tested through a series of 33 daily reversals of a light/tone successive discrimination; training was halted for 1 month and then resumed for six sessions. For the test session, the first one when training resumed, six rats relearned the last discrimination (the same correct stimulus on Sessions 33 and 34 ), but the other six rats learned the reversed discrimination (different correct stimuli on Sessions 33 and 34). The rats in the nonreversed group learned the test discrimination faster than the discrimination on Session 33, so they must have remembered the correct stimulus from Session 33, demonstrating a well established memory for the last correct stimulus in a series of discrimination reversals.

Suppose a rat is trained on a discrimination for one session, then trained on the reverse of that discrimination for a session, and so on through a series of successive reversals. Using the learning score on the original discrimination as a base rate for learning discriminations, the rate of learning of successive discrimination reversals changes systematically; learning of the first reversed discrimination is slower than the learning of the original discrimination, but, with subsequent reversals, learning of the discrimination improves. Eventually, rats will learn reversed discriminations faster than they learned the original discrimination (e.g., Dufort, Guttman, \& Kimble, 1954; Gonzalez, Berger, \& Bitterman, 1966; Mackintosh, McGonigle, Holgate, \& Vanderver, 1968).

The slower learning of the first reversed discrimination is due to negative transfer from the original discrimination; the rats remember the correct alternative from Discrimination 1 and, during the reversed discrimination, must unlearn that stimulus preference and learn a new stimulus preference. With successive reversals of the discrimination, the persistence in responding to the correct alternative for the preceding discrimination is weakened, and learning improves until the rats learn successive reversals very rapidly. Progressive improvement in serial discrimination reversals (SDR) has been attributed to accumulation of proactive interference from the previous discriminations, causing the animal to "forget" the rewarded alternative on the immediately preceding discrimination (Gonzalez et al, 1966; Woodward, Schoel, \& Bitterman, 1971; Woodward \& Bitterman, 1972). These authors argued

*Supported in part by NIMH Grant No. MH 10513 to the University of Tennessee. that proactive interference from the large number of preceding discriminations causes the animal to forget the correct alternative on the preceding discrimination, so, after experiencing a long series of serial discrimination reversals, the animal starts each new session afresh and there is no negative transfer from the immediately preceding discrimination.

Continued improvement in rate of learning successive discriminations does not prove that proactive interference accounts for it; in fact, the proactive interference interpretation of progressive improvement in SDR is ad hoc. Thus, researchers worked to develop a SDR method to isolate proactive interference effects. The best example of one is a 2-day discrimination reversal procedure, in which an animal is trained for 2 consecutive days on a discrimination, then 2 days on the reversed discrimination, and so on through several successive reversals. Day 2 of each discrimination is a nonreversal day, since the same alternative is correct, but Day 1 of each is a reversal day, since the correct alternative is changed. Negative transfer should occur on Day 1 only, and the difference in Day 1 and Day 2 scores reflects the degree of negative transfer. Early in 2-day SDR training, retention of the preference established on Day 1 of each successive reversal is good, because positive transfer from Day 1 to Day 2 is large and negative transfer from Day 2 to Day 1 is large. However, as training proceeds, both types of transfer decrease until there is very little difference in performance on Day 1 and Day 2 of successive reversals. Since both positive and negative transfer decrease, a logical deduction is that the numerous preceding reversals caused the animal to forget the preference established in the preceding session, whether it was Day 1 or Day 2 of a discrimination, and it starts each session with no bias. Thus, the type of change during 2-day SDR confirms a proactive interference interpretation of progressive improvement in SDR.

Proactive interference cannot account for all improvement in performance in SDR. If negative transfer were zero, learning of serially reversed discriminations should be equal to but not faster than the rate of learning the first discrimination, as there should be no negative transfer on the first discrimination. Yet, it is commonly observed that animals (goldfish, pigeons, rats, monkeys, and man) continue to improve with SDR until performance is better than on the first discrimination; therefore, the proactive interference explanation does not account for the full extent of progressive improvement in SDR.

This paper reports a direct test of the role of forgetting in serial discrimination reversals. Rats were first trained through a series of 33 discrimination 


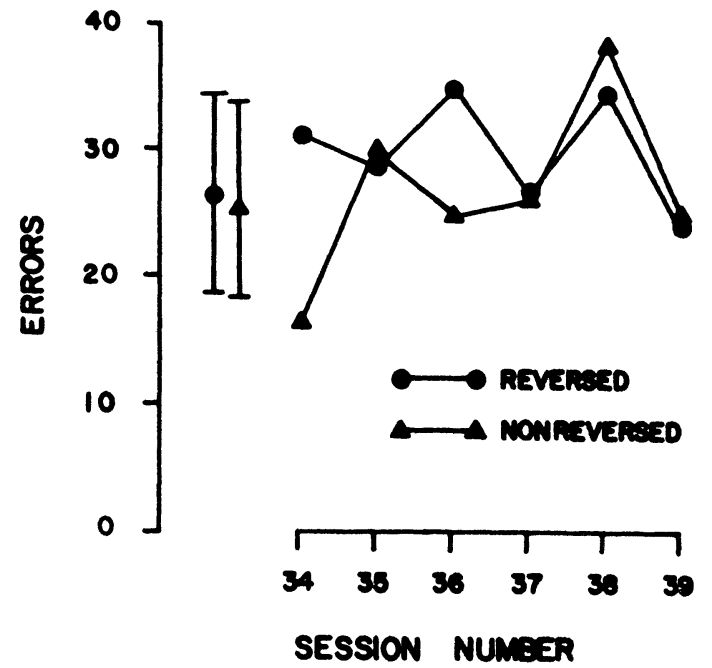

Fig. 1. Average errors per session for reversed and nonreversed groups. The bracket shows the mean $\pm 1.96 \mathrm{SE}$ for the four sessions before the training was halted; thereafter each session is plotted separately.

reversals, and then training was halted for 32 days. When training was resumed after the 32-day break, half of the rats were given the reversed discrimination and the other half the nonreversed discrimination; thereafter, SDR was continued. If the animals forgot the correct stimulus for Discrimination 33 because of proactive interference from the preceding series of reversed discriminations, we would expect the nonreversed and reversed rats to perform equally on the first postbreak discrimination session. However, if the rats remembered the last discrimination, there would be positive transfer for the nonreversed group, so that group would make fewer errors during Session 34 than during Session 33.

\section{METHOD \\ Subjects}

Twelve Sprague-Dawley rats obtained from a commercial supplier (Sprague-Dawley Company) were Ss; the rats were $200-250 \mathrm{~g}$ at the start of the original training and were approximately 85 days of age. They were maintained on a $23-\mathrm{h}$ water-deprivation schedule during the experimental periods, with free access to food. During the 30-day rest period, water was available ad lib. Three days prior to the retest, they were returned to the deprivation schedule.

\section{Apparatus}

The apparati were two identical Lafayette operant chambers, with a $61 / 2-\mathrm{W}$ red houselight in the ceiling and a $6 \frac{1}{2}-\mathrm{W}$ white cue light located directly above the lever. Tone was provided by a Sonalert signal of $2900 \mathrm{~Hz}$ in series with a $100-\mathrm{kohm}$ resister attenuating the intensity to approximately $75 \mathrm{~dB}$. Each operant chamber was enclosed in a sound-deadened cubicle during testing; a fan provided ventilation and a masking noise. Stimuli were programmed with electromechanical circuitry and data were recorded on impulse counters.

\section{Procedures}

Discrete trial operant procedures were used as described previously (Kulig \& Calhoun, 1972). A unitary SDR was used; stimuli were presented singly and there was a single response alternative (therefore, this is a go/no-go discrimination procedure). Either a tone or a light signaled a trial with a duration of $6 \mathrm{sec}$, and trials were separated by an intertrial interval (ITI) of $10 \mathrm{sec}$. Intertrial responses were suppressed by an ITI postponement; any ITI response reset the interval timer. The order of the trial stimuli was semirandom in a Gellerman series of 20 units. A single leverpress during a correct trial terminated the trial stimulus and delivered a drop of $9 \%$ sucrose; this was recorded as an S+ response. The first leverpress during an $\mathrm{S}$ - trial was counted as an error, but it did not terminate the trial and repetitive $S$ - responses were recorded.

At the start of training, the rats were randomly assigned S+, with the restriction that half received tone and half light. Each reversal consisted of one 300-trial session. SDR training was continued without interruption for 33 successive daily reversals; the rats were not tested for the next 32 days, then they were tested once more with identical procedures.

\section{RESULTS}

Data from the last four sessions prior to the break provide the baseline to evaluate performance on the test session (No. 34). The range of the group means for these four sessions is plotted in the figure. There were no differences in the two groups on any of the four baseline reversals ( $\mathrm{t}$ values ranged from +.94 to -.17 ). Session 34 was the first 300-trial session after the 32-day break in which six rats received the reversal of the last discrimination (reversed group), while the others received the same discrimination (nonreversed group). On Session 34 the nonreversed group made significantly fewer errors than the reversed group $(t=2.46, d f=1 / 10$, $\mathrm{p}<.05$, two-tailed) and significantly fewer errors than on Session 33 (correlated $t$ test, $t=2.92$, $d f=1 / 5$, $\mathrm{p}<.05$, two-tailed). However, when reversals were continued, the two groups performed quite similarly. There were no significant differences in errors for Reversals 35-39 ( $t$ tests ranged from +1.28 to -0.46 ) and, by Reversal 39, the groups performed as well as they had prior to the 32-day rest.

\section{DISCUSSION}

The most striking finding is that the rats "remembered" the last discrimination in a series of 33 serial discrimination reversals, as shown by the positive transfer for the nonreversed group from Session 33 to Session 34. Accumulated proactive interference, if present, did not interfere with this memory. It is conceivable that proactive interference dissipated over the 32-day break; however, if this were the case, the dissipation of proactive interference during the 32-day rest should cause deterioration of SDR performance for the reversed group. However, the reversed group performed as well on Reversal 34 as on Reversal 33 (means $=31.3$ and 30.8 , respectively), and with continued SDR sessions both groups performed equally well, although somewhat poorer than on Sessions 30-33.

In conclusion, the nonreversed group remembered the correct stimulus from the session immediately prior to the rest period. This experiment provides unequivocal evidence for long-term memory in the rat and questions whether proactive interference is a factor in asymptotic SDR performance.

\section{REFERENCES}

Dufort, R. H., Guttman, N., \& Kimble, G. A. One-trial discrimination reversal in the white rat. Journal of Comparative \& Physiological Psychology, 1954, 47, 248-249. 
Gonzalez, R. C., Berger, B. D., \& Bitterman, M. E. Improvement in habit-reversal as a function of amount of training per reversal and other variables. American Journal of Psychology, $1966,79,417-430$

Kulig, B. M., \& Calhoun, W. H. Enhancement of successive discrimination reversal learning by methamphetamine. Psychopharmacologia, 1972, 27, 233-240.

Mackintosh, N. J., McGonigle, B., Holgate, V., \& Vanderver, V. Factors underlying improvement in serial reversal learning. Canadian Journal o Psychology, 1968, 22, 85-95.
Woodward, T., \& Bitterman, M. E. Further studies of reversal learning with singly presented stimuli in pigeons and goldfish.

Psychonomic Science, 1972, 3, 170-172.
Woodward, T., Schoel, W. M., \& Bitterman, M. E. Reversal learning with singly presented stimuli in pigeons and goldfish. Journal of Comparative \& Physiological Psychology, 1971, 76, 460-467.

(Received for publication March 1, 1973.) 